\title{
Virtual Agents in Brain-Computer Interfaces
}

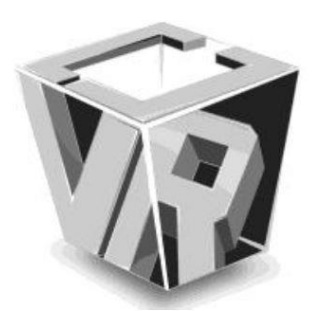

\author{
Marc Cavazza ${ }^{1}$, Fred Charles ${ }^{1}$, Stephen W. Gilroy ${ }^{1}$, Julie Porteous ${ }^{1}$, Gabor Aranyi ${ }^{1}$, \\ Julien Cordry ${ }^{1}$, Gal Raz ${ }^{2,3}$, Nimrod Jakob Keynan ${ }^{2}, 3$, Avihay Cohen², Gilan

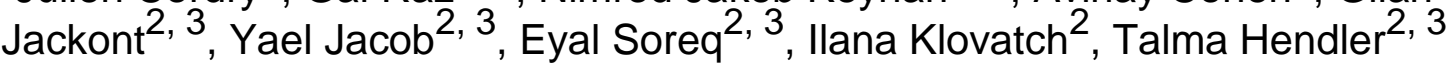 \\ ${ }^{1}$ School of Computing,Teesside University,Middlesbrough, United Kingdom,m.o.cavazza@tees.ac.uk \\ ${ }^{2}$ Functional Brain Center, Tel Aviv Sourasky, Medical Center, Tel Aviv, \\ Israel ${ }^{3}$ Tel Aviv University, Tel Aviv, Israel,hendlert@gmail.com
}

\begin{abstract}
The recent development of Brain-Computer Interfaces (BCI) for Virtual Worlds has resulted in a growing interest in realistic visual feedback. In this paper, we investigate the potential role of Virtual Agents in neurofeedback (NF) systems, which constitute an important paradigm for BCI. We discuss the potential of virtual agents to contribute to the success of NF in the specific context of affective BCI. Throughout the paper, we illustrate our presentation with two fully implemented NF prototypes featuring virtual agents. The first is an interactive narrative in which the user empathises with the feature character; the second, an emotion regulation system in which virtual crowd behaviour becomes a metaphor for arousal, as the user attempts to down-regulate their affective state.
\end{abstract}

Index Terms - Brain Computer Interfaces, Virtual Reality, Neurofeedback, Affective Computing

\section{INTRODUCTION}

In recent years, we have seen a development of synergies between Brain-Computer Interfaces (BCI) and Virtual Reality applications (Lécuyer et al., 2013). This has manifested itself in different ways, from the integration of BCI as an interface to virtual environments (Lécuyer et al., 2008) and the incorporation of BCI in computer games (Marshall et al., 2013), to the development of serious games as part of BCIbased therapeutic interventions (Lim et al., 2012; Renard et al., 2010). Virtual agents have become an important component of all the above environments, for instance in computer games and interactive narratives (Porteous et al., 2010), with their ability to express and elicit various types of emotions. This makes them of particular relevance to affective BCI (Mühl et al., 2011).

In this paper we investigate the role of virtual agents in a specific type of affective BCI system based on neurofeedback (NF). Our objective is to determine how virtual agents can be integrated into NF systems in a way that improves their usability. Previous research has suggested that the type of visual feedback used could have an influence on user experience and NF performance (Jensen et al., 2013). In the case of affective BCI, the use of virtual agents could improve the feedback channel, due to their ability to elicit emotional or social responses related to the BCI signal under consideration.

After a brief introduction to NF, and a discussion of relevant previous work, we introduce two main properties of virtual agents: their ability to enhance the subject experience; and their influence on the behaviour of NF loops. We then present two fully-implemented NF experiments featuring virtual agents. The first one implements an empathic BCI within an interactive narrative and the second one uses crowd behaviour as a feedback channel in an emotion regulation application.

\section{PRINCIPLES OF NEUROFEEDBACK}

$\mathrm{NF}$ is the process by which subjects learn to control the activation of specific brain regions in a closed-loop setting, where they are presented with a measure of that region's activation. Recent progress has provided more rigorous foundations to NF (Gruzelier, 2013), which has been proposed for performance enhancement (Nan et al., 2012) and as a therapy for several conditions (Niv, 2013), including mood disorders (Baehr et al., 2011) and ADHD (Lim et al., 2012).

$\mathrm{NF}$ has also been introduced as one possible mechanism for BCI, as it provides a more stable 
input than other measures, and preserves the user's agency. Further, its feedback component can naturally be integrated into the interface, in particular as it is most often visual. A NF system can be described through several essential components. The first one consists in the target brain area to be regulated which is generally identified through its support for specific (e.g. executive) functions, or its role in cognitive processes. The second is the sensing device, which captures that region's activation

(Weiskopf, 20112), based on various techniques of different spatio-temporal resolutions (EEG, fMRI, fNIRS...). The third one is the feedback channel, which translates the neural activity measured by the sensor into real-time information for the user. The feedback channel has as its objective the reward of desired behaviour, by notifying the user when the target region's activity is within the target range.

Another important element of any NF experiment consists in the cognitive strategies that the user will apply to control the activation of the target region (Kober et al., 2013; Nan et al., 2012). These strategies account for the fact that NF aims precisely to bring under conspicuous control neural structures that are ordinarily not amenable to it. It is customary to differentiate between explicit and implicit strategies (Sulzer et al., 2013): the former provide guidance to the user on thought contents that may result in activating the target region, while the latter leave the discovery to the user.

The development of multimedia systems and VR has influenced the design of NF systems, which can take advantage of the improved display to design more sophisticated visual feedback (see Sulzer et al. (2013) for some examples). This also makes it possible to embed NF into a realistic task: the use of virtual agents falls into this category, and will be developed in the next sections.

\section{PREVIOUS AND RELATED WORK}

The type of visual feedback used in NF systems tends to differ depending on the context of use and the nature of the system. We can distinguish three main contexts: fundamental research in neuroscience (Zotev et al., 2014), clinical applications of NF (Rosenfeld et al., 1995), and use of NF to support BCI applications. Research in NF has traditionally used basic visual (or aural) modalities for feedback, possibly because the need to engage users was felt less strongly with volunteers than patients.

Several authors have explored the use of NF systems as a therapy for ADHD, and many prototypes have been developed, of increasing complexity. Since these systems target children there has been additional consideration into the acceptability and compelling nature of the visual interface ( $\mathrm{Lim}$ et al., 2012). Independently of the generic interest in serious gaming, the familiarity of the user population with computer games has made them a natural medium for the development of NF systems. A NF system for ADHD therapy has been developed as part of the OpenVIBE project (Lécuyer et al., 2008). In this system, the user is immersed in a regular classroom setting in firstperson mode, populated with virtual characters representing other pupils. The user's perspective is one of sitting at their desk in a normal classroom, facing the blackboard. NF takes place through two main mechanisms. The first one consists in blurring the image of a virtual TV screen situated inside the classroom. However, the main feedback mechanism consists in seeing supplies on the pupil's desk levitating when the user fails to meet the activity threshold. The visual feedback hence consists in the intensity of this levitation, which is equivalent to the motion parameters of a virtual agent.

The third category consists in BCI that have been specifically developed for multimedia applications and therefore inherit the visual richness of the embedding application. One particular case is constituted by BCI to computer games, which have attracted significant interest in recent years (Marshall et al., 2013). For instance, in "alpha World of Warcraft" (Bos et al., 2010), the level of alpha activity detected from the user is used to transform the appearance of the player character between the various ones defined in the game.

Finally, Jensen et al. (2013) conducted one of the first specific studies on the impact of visual feedback in NF applications from an engineering perspective. They compared different levels of sophistication and integrative properties of the visual interface on a task of alpha-activation NF, which is known to be relevant in terms of cognitive performance enhancement (Zoefel et al., 2011). 


\section{THE REALISM ARGUMENT IN NEUROFEEDBACK}

In contrast to other variants of feedback previously reported in NF, such as gauges, thermometers (Caria et al., 2007) and colour blocks (Huster et al., 2013), the initial and naive rationale for the use of virtual agents derives from an attempt at providing realistic visuals. However, this notion of realism needs to be clarified, regardless of any intrinsic limitations due to uncanny effects. We will consider that realistic effects are split across several dimensions, which include task realism, social realism and affective realism. We shall only discuss the latter two, since the present research does not incorporate task learning or simulation.

When considering NF experiments designed primarily around emotional responses and emotion regulation, recourse to virtual characters, unlike symbolic feedback displays, are relevant to the entire NF loop: they represent a value for the variable the user is trying to regulate, while their baseline state is able to induce emotion as well. This sheds a particular light on the NF process itself since positive or negative trends may be further amplified by the direct impact of characters' appearance on the very variables that the user is trying to control. An abstract representation, such as a gauge or thermometer, does convey abstract feedback on user performance: this may impact on the users' internal state through their attentional mechanisms, but in a way which largely ignores the natural meaning of the situation represented.

Social realism can be described as the truthful representation of a real-world social situation known to elicit emotional reactions from participants or spectators alike. Social situations can elicit user emotions, as a participant through appraisal mechanisms but also as an observer. The latter is of particular interest in conjunction with some potential neural mechanisms (Blair, 2007). Affective realism has to do with emotions perceived from the characters themselves. Modern animation techniques, now available in generic software such as game engines, support a level of facial animation compatible with the expression of easily recognised emotions, which can be further enhanced by associated body attitudes. Transitioning between facial expressions can also be implemented as a NF channel (see below): whenever the application embedding NF involves affective rapport with the agent, using agent expressions as a feedback channel is a natural way to design a consistent control loop.

One topic which has attracted little attention so far is the relationship between visual realism and the subject's cognitive strategies for NF. It seems logical to consider that whenever a realistic character is used for NF, it could influence the user's cognitive strategies and bias them towards either rapport or communication with that virtual character. Few works have documented the user's declared strategies to sustain NF to the exception of (Kober et al., 2013) and (Nan et al., 2012). User debriefing following our own experiments (Cavazza et al., 2014a; Gilroy et al., 2013) appears consistent with some of the strategies previously reported and, despite the small scale of our sample, a significant proportion of users made reference to communicating with virtual characters in both applications reported below. 


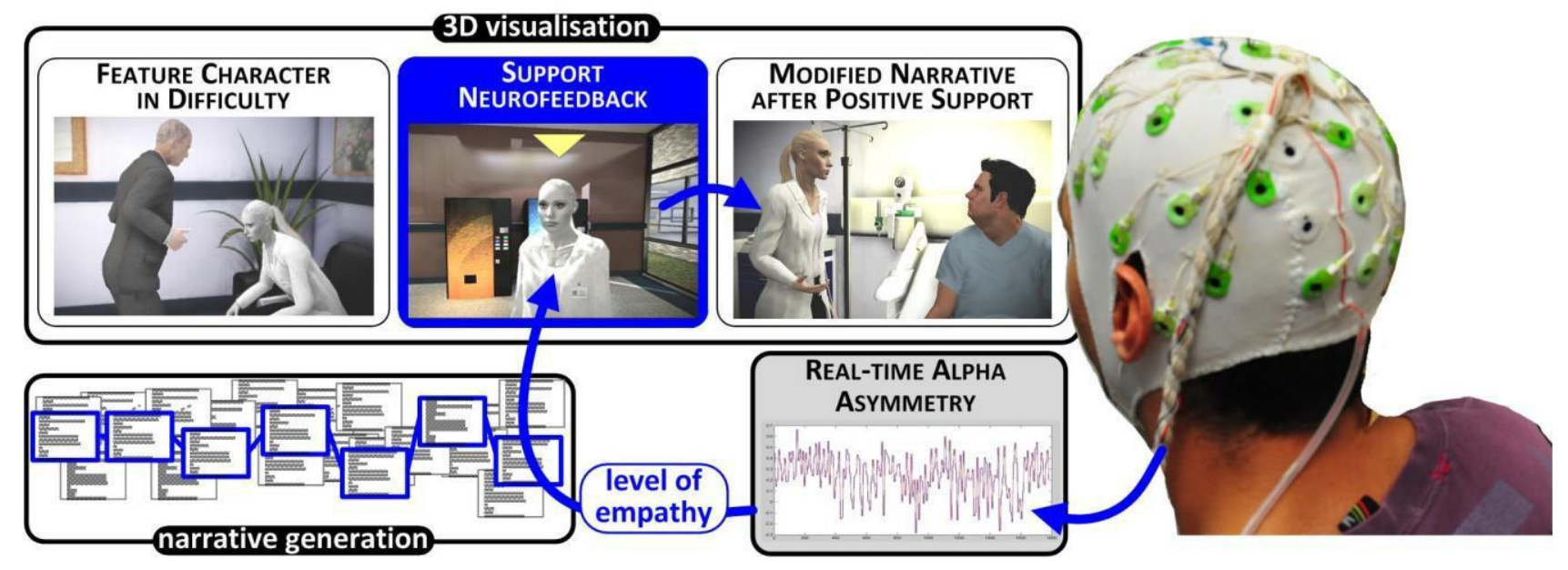

Figure 1. System Overview and Experimental Setting: the user watches the 3D narrative generated in real-time; BCI input (prefrontal alpha asymmetry) during NF sessions is interpreted in terms of empathy towards the feature character.

\section{EMPATHIC INTERACTION WITH VIRTUAL ACTORS}

An important area of research in virtual agents has been the study of relationships between humans and agents, in particular how human users respond to agents characteristics, perceived for instance as personality traits. Gratch et al. (2007) have discussed rapport, and de Melo et al. (2009) have discussed moral emotions with virtual agents. Morrison and Ziemke (2005) have discussed the conditions under which computer games' characters can elicit empathic reactions from humans: their perspective was very much a motor one, in line with the hypothesised role of mirror neurons in certain types of empathy. This body of work suggests that agents can elicit attachment, which should make users respond to further real-time evolution of an agent's situation, and/or the expression by the agent of its response to it. From a different perspective, research in filmic theory of emotions has suggested that empathy towards (human) feature characters was a key determinant of viewers' affective response (Tan, 1996).

In earlier work we explored the use of an "empathic BCI" for Interactive Narratives featuring virtual actors (Gilroy et al., 2013). Our objectives were to improve affective interaction in virtual storytelling to incorporate hypotheses from affective filmic theories (Tan, 1996), as we had previously explored passive detection of emotional response (Gilroy et al., 2012) and active speech-based emotional input (Cavazza et al., 2009). This experiment was also an effort in unifying the different components of the interactive narrative experience around the concept of empathy: narrative generation is geared towards creating difficult situations for the feature character; empathy then in turn determines the user's emotional response to that situation, and the BCI input attempts to detect user support, using brain signals previously associated with empathy (Light et al., 2009) and also amenable to NF (Baehr et al., 2001; Davidson, 2004).

The baseline story is adapted from the medical drama genre and features a female junior doctor facing multiple difficulties in her professional life. We have previously used the same setting to explore affective response through physiological sensing (Gilroy et al., 2012), as the genre is prone to elicit a wide range of affective responses based on the medical situations themselves (visuals and tension), as well as the characters' relationships. The system's architecture is represented in Figure 1. Visual generation and staging takes the form of a machinima-like 3D animation, and is based on the Unreal $^{\circledR}$ (UDK) game engine, which includes support for expressive animations and camera placement. This allows a significant level of dramatisation and enhances the potential for empathising with the main character. A baseline set of narrative actions has been defined following the traditional conventions of the medical drama genre: typically one episode will intertwine medical challenges (solving a difficult clinical case) and strained relationships with colleagues, friends and management. Each action involving or 
affecting the feature character is categorised according to the level of hardship it is placing on her. Narrative generation is based on landmark planning (Porteous et al., 2010) and is

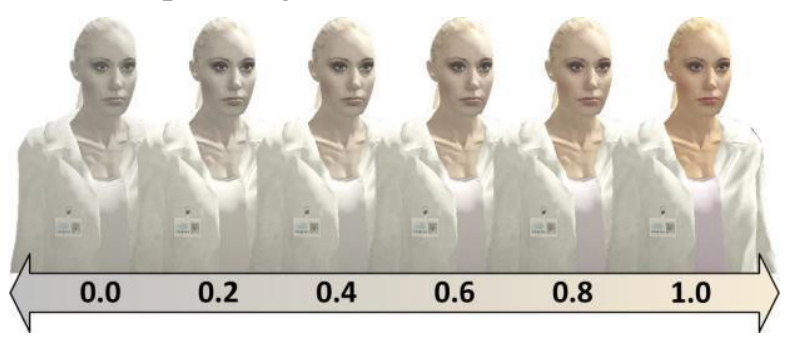

Figure 2. Feedback channel for the filmic empathy experiment. When in need of support, the feature character would fade to grey. During NF, alpha asymmetry scores are mapped onto the character's colour saturation.

biased towards placing the character through difficult situations. Although the system will generate a range of different stories, they all have story endings leading to her demise (e.g. medical malpractice, breakdown, etc.), as the story builds up adverse situations and difficulties. The system is biased towards creating difficult situations at an early stage (first third of the story). Once the character is faced with a serious situation, she will turn to the user for support. This manifests itself through an embedded NF session, the user having been briefed about the possibility of helping her by "expressing positive thoughts".

Should the user succeed in supporting her, the remainder of the narrative will evolve in a direction more favourable to the character. The user is given between one and two opportunities for interaction, which take the form of short $30 \mathrm{~s}$ $\mathrm{NF}$ sessions. The NF technique consists in a measure of pre-frontal alpha EEG asymmetry using Davidson's A2 score (Davidson, 1992). This measure is correlated with approach/avoidance, as well as a specific kind of empathy described as cheerful empathy (Light et al., 2009).

The NF session is triggered by interrupting the narrative action and transitioning to a cut scene in which the character stands alone in the hospital corridor, facing the user. The level of desperation of the character is visualised by desaturating her colour until she appears in black and white within an otherwise colour scene (Figure 2). Another justification to isolate the NF session is the hypothesised difficulty for users to sustain the BCI input by altering their pre-frontal activation. The role of the virtual character in the NF process is thus double: it elicits empathic responses by expressing her disarray (Figure 3) and it serves as the output signal for NF.

We carried out three sets of experiments using alpha asymmetry NF as an affective BCI to the Interactive Narrative. In all experiments, it was explained to subjects that the feature character in the narrative will face difficulties and that they had the power to help her using the BCI. Subjects were introduced to the interactive narrative and was explained that they would be able to influence the story by supporting the feature character "through positive thinking", without being given explicit instruc-

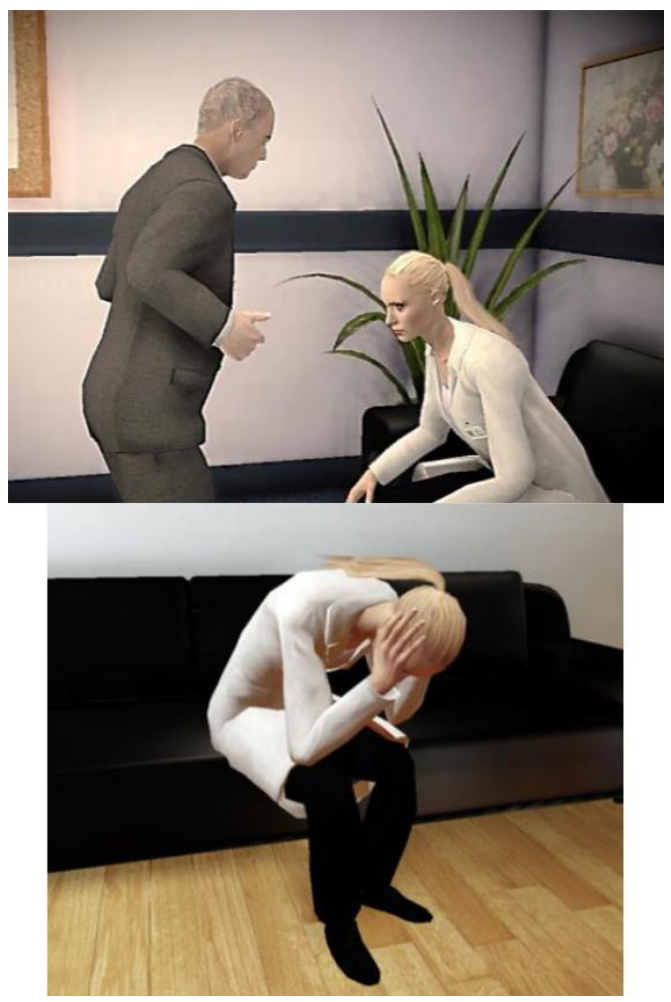

Figure 3. Narrative events showing the feature character in a difficult situation. 


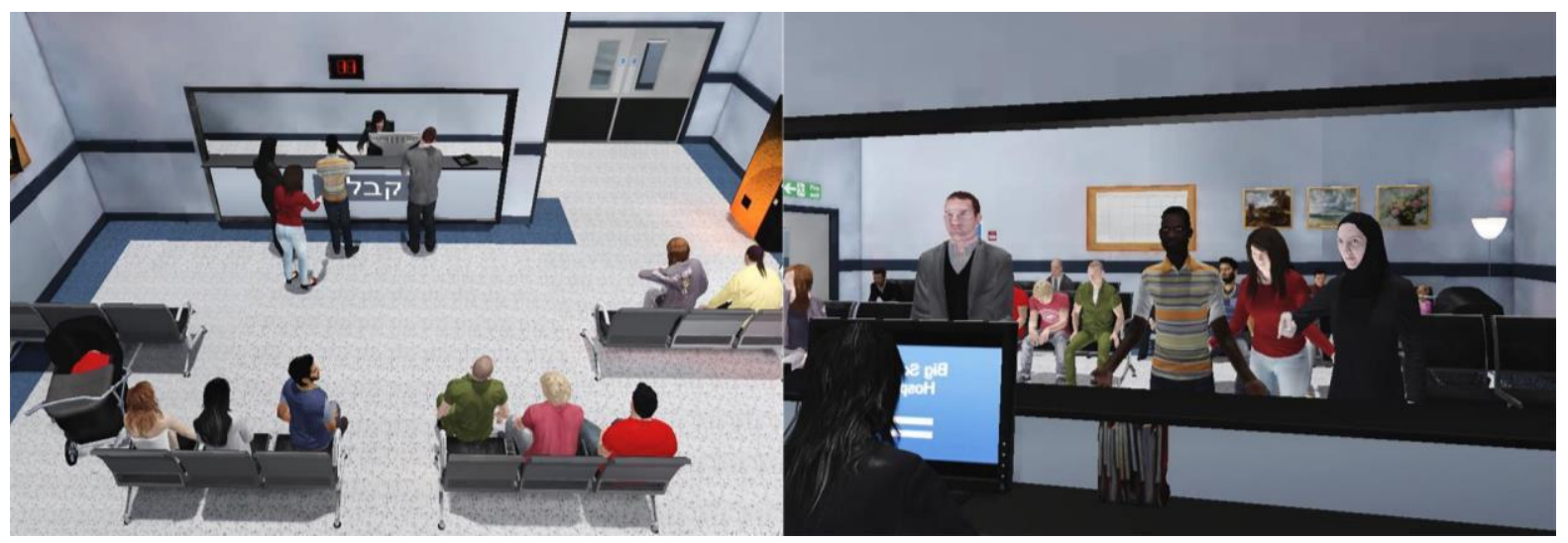

Figure 4. Overview of the Waiting Room environment.

tions. They were familiarised with the coloursaturation NF approach through short training sessions outside the MRI scanner. All experiments used the same interactive narrative generation system based on our previous system (Gilroy et al., 2012) and the same input signal $\left(\mathrm{A}_{2}\right.$ asymmetry score in the alpha band (8$12 \mathrm{~Hz})$ measured from $F_{3}(L)$ and $F_{4}(R)$ electrodes and defined as $\left.\left(F_{4}-F_{3}\right) /\left(F_{4}+F_{3}\right)\right)$. The experiments differed in their objectives and progressively refined the approach in terms of interaction design and real-time signal analysis.

In our first proof-of-concept experiment, subjects interacted with the system inside an fMRI scanner (using an MRI-compatible EEG device/recorder): the objective was to provide an empirical validation, suggesting that successful NF sessions were indeed associated to an asymmetry in pre-frontal areas. Out of twelve subjects, six were successful in their NF session and in altering the course of action of the narrative. From a brain imaging perspective, successful subjects showed various levels of asymmetry in the pre-frontal cortex, compared to a control condition that consisted in passively viewing a replay of the generated story without NF. No differences were observed in other areas, in particular motor areas (Gilroy et al., 2013). These findings were all compatible with our initial hypothesis. The $50 \%$ success score was somehow below typical BCI performance, but this can be explained by the very limited amount of training given to subjects (one preexperiment session of 10 minutes, compared to repeated sessions totalling several hours for most pre-frontal alpha-asymmetry reported work), as well as the MRI scanner being a difficult environment for the subjects. Our second experiment was carried out in a normal laboratory setting using the same BCI Interactive Narrative with two notable modifications: i) subjects were only given one opportunity to interact with the narrative (compared to two in the EEG-fMRI experiment) and ii) the NF success score was redefined to better account for individual differences in $\mathrm{A}_{2}$ baseline values, resulting in a slightly more demanding success criterion. This resulted in a global performance of $52.7 \%$ on a set of 36 subjects, but also uncovered a possible negative correlation between the $\mathrm{A}_{2}$ baseline and $\mathrm{NF}$ success. This was found of particular significance, since we measured the $\mathrm{A}_{2}$ baseline by alternating epochs of open eyes and closed eyes recording. This method, which was inspired from early alpha-asymmetry NF work (Rosenfeld et al., 1995) was found to overestimate the $\mathrm{A}_{2}$ baseline by 0.1 compared to open eyes recordings only (Cavazza et al., 2014b), almost half of the expected A2 variation range. We thus carried out a last experiment with 9 subjects using only eyes open recording to measure the $\mathrm{A}_{2}$ baseline, which is consistent with Davidson (1992) experiments on alphaasymmetry responses to films. All other parameters were unchanged since the second experiment and the overall success rate was this time $67 \%$, which is now in line with previous $\mathrm{BCI}$ experiments.

From a narrative perspective, the NF scene is only partly diegetic: it shares the same character and takes place within the same environment, but on the other hand requires a pause in the narrative flow. The choice of such non-diegetic feedback was initially dictated by the need to isolate the NF sessions within the course of the interactive narrative, taking into account the cognitive effort required from the subject to engage in NF and minimising other artefacts 
potentially induced by the narrative (including, but not limited to, optical flow). Our initial choice was towards a visual mapping philosophy, which supported continuous quantifiable feedback. Yet, despite the positive results obt-
One less developed area of research is to consider that populations of virtual agents, or virtual crowds, rather than individuals, can be used for the purpose of emotion visualisation, and possibly elicitation (McHugh et al., 2010). These two aspects (emotion visualisation and

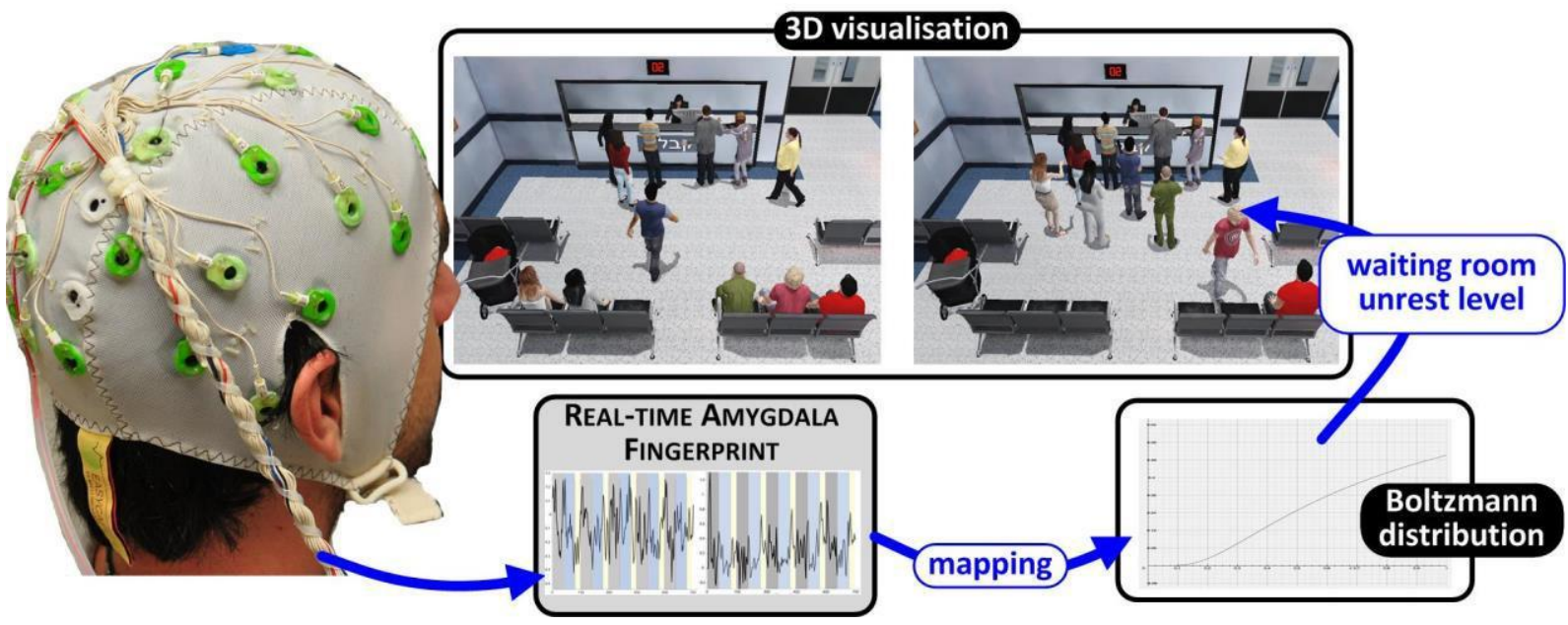

Figure 5. System Overview and Experimental Setting: the user is instructed to appease the situation in the waiting room. NF is based on a bespoke EEG Fingerprint (EFP) for the amygdala: the level of unrest in the waiting room matches the subject's amygdala's activity.

ained during the above experiments, one criticism sometimes made by subjects mentioned the somehow unrealistic nature of the NF setting. Any further improvement should not compromise the balance between the cognitive effort demanded by NF and the attentional impact of the narrative. Potential improvements can target the setting and the transitioning from the main narrative action, or use more realistic visual input, such as character's non-verbal behaviour, without jeopardising the ability to produce continuous feedback that can be mapped onto the $\mathrm{A}_{2}$ value.

\section{VIRTUAL CROWDS AND EMOTION REGULATION}

In the previous section, we have reported how a single virtual character could be used as a visual channel for NF when considering emotions elicited by the agent itself as part of the user's affective response to a narrative. This was appropriate to filmic emotions that fall within the general concept of empathy, which requires a character as a "target" to experience empathy. elicitation) tend to follow a different logic for single agents and for crowds. For instance, the visualisation of the subject's arousal through a crowd agitation level can be metaphorical, as there is no pre-established bond between the crowd and the subject. The effect of the crowd agitation on the level of user arousal is also more difficult to establish than in the case of empathic phenomena.

In a second set of experiments, reported here, we explored crowd behaviour as a potential backchannel for NF. The objective of this research was to explore the impact of realistic, as opposed to abstract, visual feedback in the context of basic research in fMRI or EEG-based NF. While our previous work was dedicated to incorporating NF-based BCI into an interactive storytelling system, this one is devoid of any media component, and was instead designed to support NF research.

It is not sufficient for a virtual crowd simulation to be realistic in terms of global behaviour; it also needs to correspond to a believable situation. We have thus designed a virtual environment in which a population of agents could rapidly evolve between emotional states in a realistic context. The situation recreated is the waiting room of a hospital, populated by twenty virtual agents playing the role of patients or family members (Figure 4). These characters can take one of two states: the default, resting state, where they sit waiting, or an excited state in which they walk up to the front desk to complain about the wait. However, the purpose 
of the environment is to convey a global sense of arousal through the agitation of the crowd, represented by the number of characters protesting at the front desk. In other words, the distribution of characters between the resting state (sitting in the waiting area) and the excited state (standing at the desk) constitutes a virtual allegory of arousal.

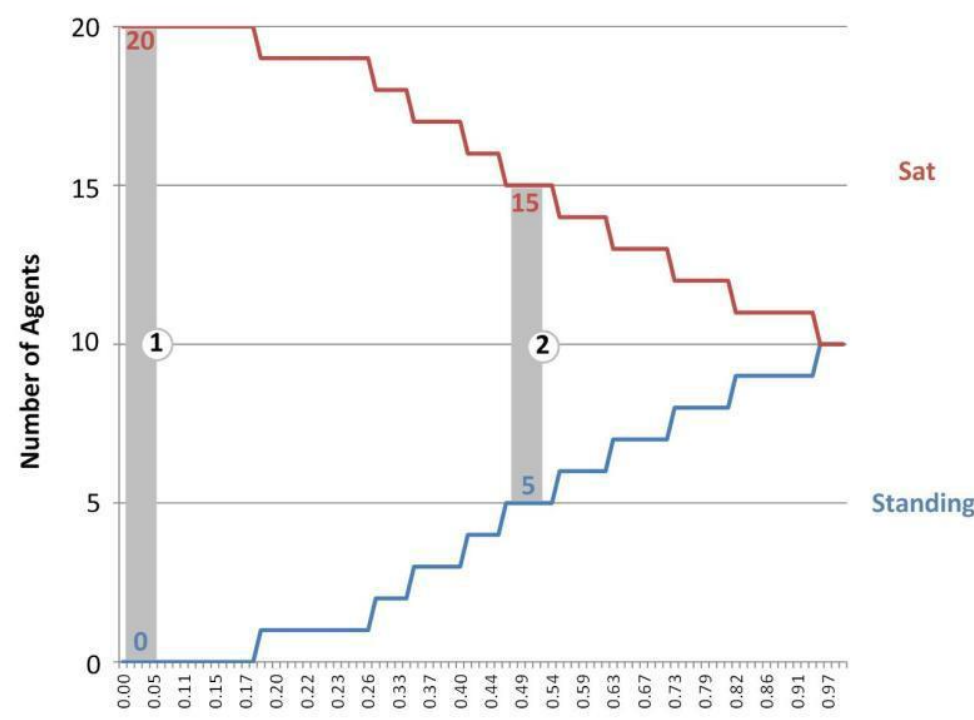

Figure 6. Evolution of the characters' distribution between states as a function of amygdala's activity for a total number of $\mathbf{2 0}$ characters.

This second NF experiment is about emotional regulation and more-specifically the downregulation of the amygdala, whose activation is generally associated with arousal (Lindquist et al., 2012). Hence the "virtual" arousal of the crowd, or level of unrest, which is the matching variable, is used to visualise the real arousal level of the subject, while they try to control their amygdala's activation.

This experiment (Figure 5) is also based on a NF paradigm, and comprises a target emotional response (arousal), a target brain region corresponding to that emotional response (amygdala), an input probe (specific EEG input known as "fingerprint"), and a visual backchannel that can be mapped onto the emotional response (the level of unrest in the waiting room). The overall experiment investigates the control of arousal based on down-regulation of the amygdala. Once again, appropriate user instructions are issued so the user can follow the NF paradigm: these

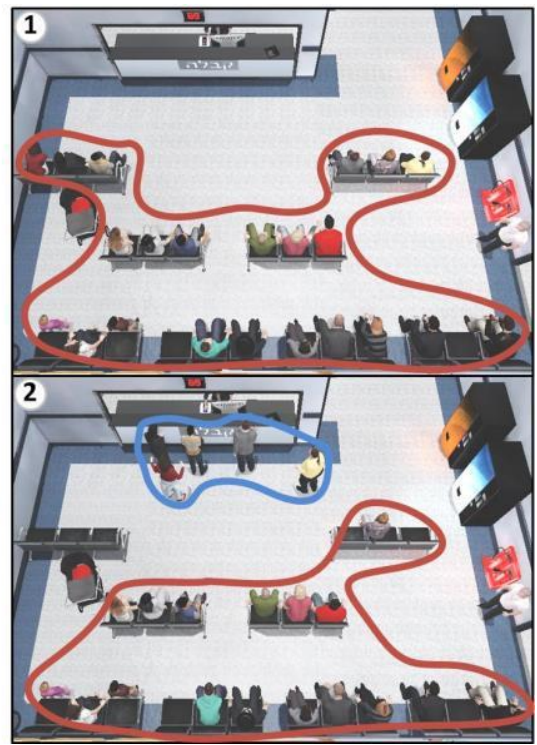

2013). In terms of signal acquisition, it brings

instructions are deliberately generic so as to avoid influencing cognitive NF strategies ("try to appease the situation in the waiting room").

The sensor is a bespoke EEG fingerprint (EFP) of the amygdala activity obtained from fMRI data, consisting in a nonstandard electrode placement as described in (Meir-Hasson et al.,

flexibility, ease of use and anatomical specificity. It also allows for a response time which is that of the EEG signal, making it possible to design appropriate feedback loops not limited by signal acquisition delays.

The system is implemented using the Unreal ${ }^{\circledR}$

(UDK) game engine: agents' behaviours are represented through various features of the engine: idle animations, motion planning and expressive animations. Each corresponds to a different component of crowd expressivity. Idle animations are required to ensure realism of the sat down fraction of the agent population, yet its parameterisation can convey a first level of unrest amongst the crowd. Motion planning, including corresponding animations is the main mechanism for crowd behaviour, in that it controls the ratio between characters waiting and characters standing at the front desk. Expressive animations that correspond to argumentative conversation further enhance the realism of the standing part of the crowd. In addition, the sound features of the Unreal ${ }^{\circledR}$ (UDK) game engine have been used to playback a global soundtrack in the form of loud conversations, whose intensity can be tuned to the unrest level of the crowd. 
As part of the NF loop, we need to be able to consistently control the overall behaviour of the crowd with a global variable. To that effect, we consider the global crowd behaviour to be described by the distribution between resting and agitated characters, which is best described by a statistical model. By analogy with statistical models describing the transition of particles between energy levels, we use a Boltzmann-type distribution for which the two levels of energy correspond to characters resting, or protesting at the front desk (Figure $6)$. The controlling variable is represented by counter or, conversely returning to their initial seat. While the change in state for an individual agent requires a few seconds to be completed (depending on its distance from the front desk), the directions in which agents move, is clearly visible.

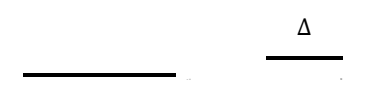

A preliminary study included 9 subjects with at least 12 years of education. Due to technical

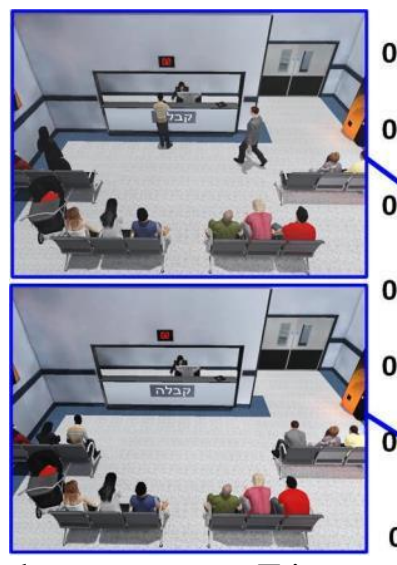

the temperature $\mathrm{T}$ in
Boltzmann's equation (see eq. 1), which is used to determine the global level of unrest in the waiting room. The NF mapping is defined by using the measured amygdala activity as an analogue of temperature in the Boltzmann equation. The other parameters in the equation, such as energy levels, have been determined empirically by a calibration step, which adapts the target distribution of agents to the variation range of the amygdala EFP input signal. In terms of NF cycles, at each step the EFP signal is acquired and converted into a virtual temperature in the Boltzmann equation. This is used to update accordingly the ratio between quiet and agitated characters, which is then adjusted in the waiting room by computing the required adjustment between number of characters in each state, and randomly selecting agents for transition (Figure 7). Transitioning from states is visualised in terms of the agent standing up from its seat and approaching the

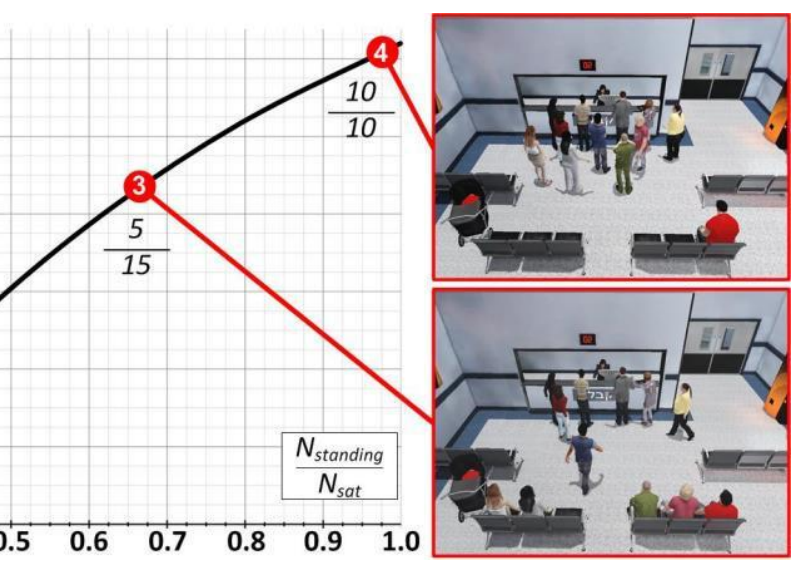

problems the data sets for one subject were discarded. Thus, overall, 8 subjects (age: $25.5 \pm$ 1.66; 3 females) were included in the analysis.

EFP data was collected under three separate blocks (see Figure 8): (i) Finger tapping task (30s) during which participants were instructed to tap a finger three times according to a preset stimulus. This task was used to "reset" the EFP level following a NF epoch and before a baseline epoch. (ii) Baseline condition (60s) during which the subject passively viewed the Waiting Room. A fixed unrest level of 0.5 was set during this epoch. (iii) NF task (60s) during which the subject was instructed to relax the waiting room.

Each subject went through two sessions started with conditions (ii) and (iii), and followed by either two or four repetitions of the triplet (i)(ii)-(iii). The only exception was a single subject who due to technical problems was trained with two baseline and NF epochs (both of 180s long) during the first session and two $\mathrm{NF}$ and baseline conditions (60s both) during the second. Thus, each subject was trained with 8 baseline and NF pairs in total.

EEG was recorded using an 8-channel Brain Products V-Amp system. The signal was 
analysed online using Brain Products' BrainVision RecView and our bespoke analysis software. For each point of measurement in the $\mathrm{NF}$ epoch, a value between 0 and 1 was computed and used for further computation of unrest levels as described in equation (see eq. 2 ). The NF value was computed relative

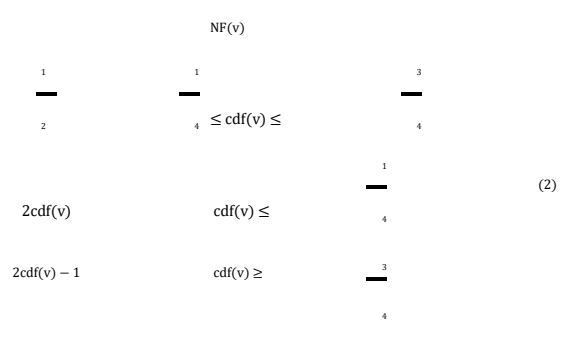

to the preceding baseline epoch representing the two-tailed probability of achieving this value or greater in both extremes as follows:

Where $v$ is the EFP sample during the feedback and $c d f(v)$ is the Cumulative Density Function of the normalized EFP samples during the baseline epoch.

The success in down-regulating the EFP during $\mathrm{NF}$ epochs, relative to the previous baseline condition was assessed by applying student's ttest to compare EFP values in successive baseline-NF epochs. This analysis yielded a tstatistic for each baseline-NF duplet. A Wilcoxon signed-rank test was subsequently used to estimate EFP differences in two ways:

(a) Fixed effect - Wilcoxon test was applied using the entire population of the t-statistics. (b) Random effect - Wilcoxon test was applied on the set of medians of each subject's t-statistics.

Both the fixed and random effect analyses indicated that the subjects significantly decreased the amygdala EFP during the NF epoch $(z=-2.82, p<0.005 ; z=-2.52, p<0.008$, respectively) in line with the instructions they were given. A percent change of the EFP was calculated by summing up the differences between each of the NF samples and the corresponding average EFP baseline level divided by the number of NF samples. The median value of the EFP percent change was $28.65 \%$, which constitutes a notable variation, even more so down-regulating from a nearresting baseline (Figure 9).

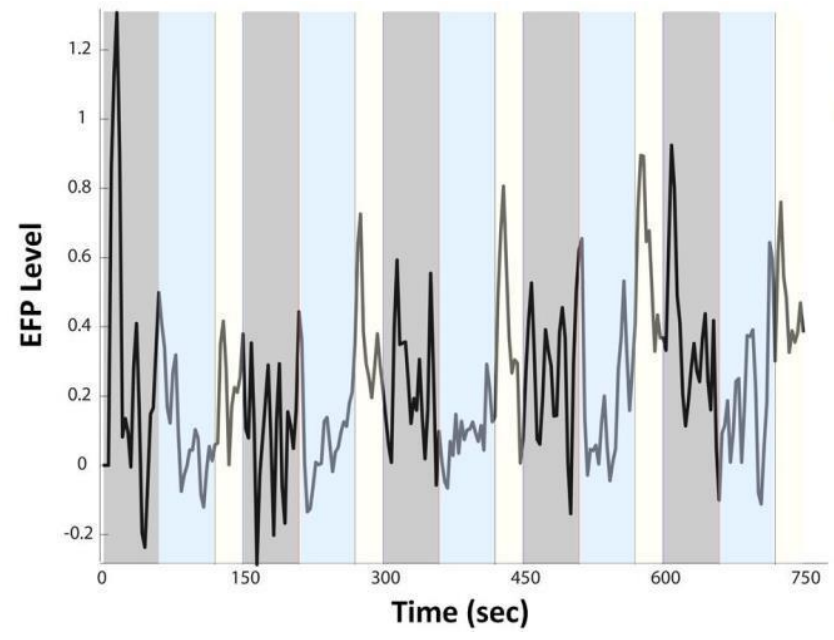

Baseline

Neurofe

Finger $t$

Figure 8. Amygdala EFP signal during an NF session in which the subject was instructed to down-regulate.

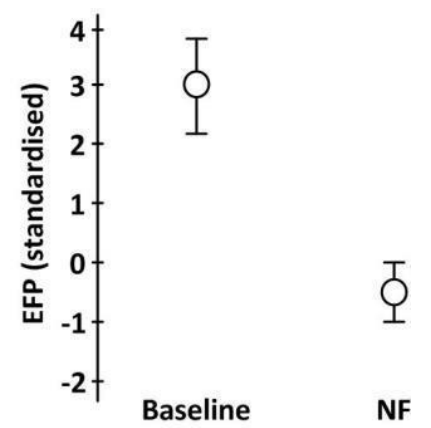

Figure 9: Mean EFP levels during baseline and NF epochs. In each session and epoch, the EFP was first standardised by dividing it by the average signal of the entire session.

\section{CONCLUSION}

An increasing number of BCI systems are turning to virtual agents as an interface for displaying NF signals. Depending on the main focus of the application, agents can: i) support various metaphors (such as the pole vault jump of (Heinrich et al., 2004), ii) participate in the realism of the situation that the subjects are training for (e.g. a classroom in ADHD treatment applications), or iii) constitute the main target of the application (e.g. in gaming or interactive narrative).

It is probably too early to draw generic conclusions about the potential benefits of virtual agents, outside those applications where they already feature prior to the adoption of BCI techniques. However, our preliminary results suggest that virtual agents can successfully 
support NF applications in affective BCI or emotional regulation experiments. This extends the research programme introduced in (Jensen et al., 2013), which suggested that more sophisticated visualisation could improve NF performance. On the other hand, this is somehow in contradiction with the previous assumption that NF should be based on a simple and direct representation of the target parameters' values (Nan et al., 2012).

More systematic research is required to compare the usability of NF systems using abstract displays with those featuring virtual agents, exploring important aspects such as temporal behaviour, ease of training and its impact on BCI illiteracy scores as in Vidaurre and Blankertz (2010).

\section{REFERENCES}

Baehr, E., Rosenfeld, J.P., and Baehr, R. 2001. Clinical use of an alpha asymmetry neurofeedback protocol in the treatment of mood disorders: Follow-up study one to five years post therapy. Journal of Neurotherapy 4, 4, 11-18.

Blair, R. 2007. The amygdala and ventromedial prefrontal cortex in morality and psychopathy. Trends in Cognitive Sciences 11, 9, 387-392.

Bos, D.P., Reuderink, B., van de Laar, B., Gürkök, H., Mühl, C., Poel, M., Nijholt, A., and Heylen, D. 2010. Brain-computer interfacing and games. In Brain-Computer Interfaces, D. Tan and A. Nijholt, Eds. Springer, 149-178.

Caria, A., Veit, R., Sitaram, R., Lotze, M., Weiskopf, N., Grodd, W., and Birbaumer, N. 2007. Regulation of anterior insular cortex activity using real-time fMRI. NeuroImage $35,3,1238-1246$.

Cavazza, M., Pizzi, D., Charles, F., Vogt, T., and André, E. 2009. Emotional input for character-based interactive storytelling. In

Proceedings of the 8th International Conference on Autonomous Agents and Multiagent SystemsVolume 1, International Foundation for Autonomous Agents and Multiagent Systems, 313-320.

Cavazza, M., Aranyi, G., Charles, F., Porteous, J., Gilroy, S., Klovatch, I., Jackont, G., Soreq, E., Keynan, N. J., Cohen, A., Raz, G. and Hendler, T. 2014a. Towards Empathic Neurofeedback for Interactive Storytelling. In Proceedings of 2014 Workshop on
Computational Models of Narrative (CMN 2014), July 31-August 2, Quebec City, Canada. (to appear)

Cavazza, M., Aranyi, G., Charles, F., Porteous, J., Gilroy, S., Jackont, G., Klovatch, I., Raz, G., Keynan, N.J., Cohen, A., and Hendler, T.

2014b. Frontal Alpha Asymmetry

Neurofeedback for Brain-Computer Interfaces. In Proceedings of the 6th International BrainComputer Interface Conference 2014, Graz, Austria, September 16-19. (to appear)

Davidson, R.J. 1992. Anterior cerebral asymmetry and the nature of emotion. Brain and Cognition 20, 1, 125-151.

Davidson, R.J. 2004. What does the prefrontal cortex "do" in affect: perspectives on frontal EEG asymmetry research. Biological Psychology 67, 1, 219-234.

de Melo, C.M., Zheng, L., and Gratch, J. 2009. Expression of moral emotions in cooperating agents. In Proceedings of the 9th International Conference on Intelligent Virtual Agents (IVA), Zs. Ruttkay, M. Kipp, A. Nijholt, H. Vilhjálmsson, Eds. Springer, 301-307.

Gilroy, S., Porteous, J., Charles, F., and Cavazza, M. 2012. Exploring passive user interaction for adaptive narratives. In

Proceedings of the 2012 ACM international conference on Intelligent User Interfaces, ACM, 119-128.

Gilroy, S.W., Porteous, J., Charles, F., Cavazza, M., Soreq, E., Raz, G., Ikar, L., OrBorichov, A., Ben-Arie, U., and Klovatch, I. 2013. A brain-computer interface to a planbased narrative. In Proceedings of the TwentyThird International Joint Conference on Artificial Intelligence, AAAI Press, 1997-2005.

Gratch, J., Wang, N., Gerten, J., Fast, E., and Duffy, R. 2007. Creating rapport with virtual agents. In Proceedings of the 7th International Conference on Intelligent Virtual Agents (IVA), C. Pelachaud, J-C. Martin, E. André, G. Chollet, K. Karpouzis, D. Pelé, Eds. Springer, 125-138.

Gruzelier, J.H. 2013. EEG-neurofeedback for optimising performance. I: A review of cognitive and affective outcome in healthy participants. Neuroscience \& Biobehavioral Reviews.

Heinrich, H., Gevensleben, H., Freisleder, F.J., Moll, G.H., and Rothenberger, A. 2004.

Training of slow cortical potentials in attentiondeficit/hyperactivity disorder: evidence for 
positive behavioral and neurophysiological effects. Biological Psychiatry 55, 7, 772-775.

Huster, R.J., Mokom, Z.N., Enriquez-Geppert, S., and Herrmann, C.S. 2013. Brain-computer interfaces for EEG neurofeedback:

Peculiarities and solutions. International Journal of Psychophysiology 91, 1, 36-45.

Jensen, C.B.F., Petersen, M.K., Larsen, J.E., Stopczynski, A., Stahlhut, C., Ivanova, M.G., Andersen, T., and Hansen, L.K. 2013. Spatio temporal media components for neurofeedback. In Proceedings of the 2013 IEEE International Conference on Multimedia and Expo (ICME).

Kober, S.E., Witte, M., Ninaus, M., Neuper, C., and Wood, G. 2013. Learning to modulate one's own brain activity: the effect of spontaneous mental strategies. Frontiers in Human Neuroscience 7, Article 695.

Lécuyer, A., Lotte, F., Reilly, R.B., Leeb, R., Hirose, M., and Slater, M. 2008. Braincomputer interfaces, virtual reality, and videogames. Computer 41, 10, 66-72.

Lécuyer, A., George, L., and Marchal, M. 2013. Toward Adaptive VR Simulators Combining Visual, Haptic, and Brain-Computer Interfaces. IEEE Computer Graphics and Applications, vol. 33, no. 5, 18-23.

Light, S.N., Coan, J.A., Zahn-Waxler, C., Frye, C., Goldsmith, H.H., and Davidson, R.J. 2009. Empathy is associated with dynamic change in prefrontal brain electrical activity during positive emotion in children. Child

Development 80, 4, 1210-1231.

Lim, C.G., Lee, T.S., Guan, C., Fung, D.S.S., Zhao, Y., Teng, S.S.W., Zhang, H., and Krishnan, K.R.R. 2012. A Brain-Computer Interface Based Attention Training Program for Treating Attention Deficit Hyperactivity Disorder. PloS one 7, 10, e46692.

Lindquist, K.A., Wager, T.D., Kober, H., BlissMoreau, E., and Barrett, L.F. 2012. The brain basis of emotion: A meta-analytic review. Behavioral and Brain Sciences 35, 3, 121-143.

Marshall, D., Coyle, D., Wilson, S., and Callaghan, M. 2013. Games, gameplay, and BCI: The state of the art. IEEE T-CIAIG 5, 2, 82-99.

McHugh, J.E., McDonnell, R., O’Sullivan, C., and Newell, F.N. 2010. Perceiving emotion in crowds: the role of dynamic body postures on the perception of emotion in crowded scenes.

Experimental Brain Research 204, 3, 361-372.
Meir-Hasson, Y., Kinreich, S., Podlipsky, I., Hendler, T., and Intrator, N. 2013. An EEG Finger-Print of fMRI deep regional activation. NeuroImage.

Morrison, I. and Ziemke, T. 2005. Empathy with computer game characters: A cognitive neuroscience perspective. In Proceedings of the Joint Symposium on Virtual Social Agents, AISB, Hatfield, U.K., 73-79.

Mühl, C., Brouwer, A., van Wouwe, N., van den Broek, E., Nijboer, F., and Heylen, D. 2011. Modality-specific affective responses and their implications for affective BCI. In Proceedings of the Fifth International BrainComputer Interface Conference, G.R. MüllerPutz, R. Scherer, M. Billinger, A. Kreilinger, V. Kaiser, C. Neuper, Eds. Verlag der Technischen Universität, Graz, Austria, 120-123.

Nan, W., Rodrigues, J.P., Ma, J., Qu, X., Wan, F., Mak, P., Mak, P.U., Vai, M.I., and Rosa, A. 2012. Individual alpha neurofeedback training effect on short term memory. International Journal of Psychophysiology 86, 1, 83-87.

Niv, S. 2013. Clinical efficacy and potential mechanisms of neurofeedback. Personality and Individual Differences 54, 6, 676-686.

Porteous, J., Cavazza, M., and Charles, F. 2010. Applying planning to interactive storytelling: Narrative control using state constraints. ACM Transactions on Intelligent Systems and Technology (TIST) 1, 2, 1-21.

Renard, Y., Lotte, F., Gibert, G., Congedo, M., Maby, E., Delannoy, V., Bertrand, O., and Lécuyer, A. 2010. OpenViBE: an open-source software platform to design, test, and use braincomputer interfaces in real and virtual environments. Presence: Teleoperators and Virtual Environments 19, 1, 35-53.

Rosenfeld, J.P., Cha, G., Blair, T., and Gotlib, I.H. 1995. Operant (biofeedback) control of left-right frontal alpha power differences: Potential neurotherapy for affective disorders. Biofeedback and Self-Regulation, 20(3):241-258.

Stoiber, N., Seguier, R., and Breton, G. 2009. Automatic design of a control interface for a synthetic face. In Proceedings of the 14th international conference on Intelligent User Interfaces, ACM, 207-216.

Sulzer, J., Haller, S., Scharnowski, F., Weiskopf, N., Birbaumer, N., Blefari, M., Bruehl, A., Cohen, L., Decharms, R., and 
Gassert, R. 2013. Real-time fMRI

neurofeedback: Progress and challenges.

NeuroImage 76, 386-399.

Tan, E.S. 1996. Emotion and the structure of narrative film: Film as an emotion machine.

Lawrence Erlbaum Associates, Mahwah, N.J.

Vidaurre, C. and Blankertz, B. 2010. Towards a cure for BCI illiteracy. Brain Topography 23, 2, 194-198.

Weiskopf, N. 2012. Real-time fMRI and its application to neurofeedback. NeuroImage 62, 2, 682-692.

Zoefel, B., Huster, R.J., and Herrmann, C.S. 2011. Neurofeedback training of the upper alpha frequency band in EEG improves cognitive performance. NeuroImage 54, 2 , 1427-1431.

Zotev, V., Phillips, R., Yuan, H., Misaki, M., and Bodurka, J. 2014. Self-regulation of human brain activity using simultaneous real-time fMRI and EEG neurofeedback. NeuroImage, 85:985-995. 